\title{
Open circuit voltage increase of GaSb/GaAs quantum ring solar cells under high hydrostatic pressure
}

\author{
D. Montesdeoca ${ }^{1}$, P. J. Carrington ${ }^{2}$, I. P. Marko ${ }^{3}$, M. C. Wagener ${ }^{4}$, S. J. Sweeney ${ }^{3}$ and A. \\ Krier $^{1}$ \\ ${ }^{1}$ Physics Department, Lancaster University, Lancaster, LA1 4YB, UK \\ ${ }^{2}$ Engineering Department, Lancaster University, Lancaster, LA1 4YW, UK \\ ${ }^{3}$ Physics Department and Advanced Technology Institute, University of Surrey, Guildford, \\ Surrey, GU2 7XH, UK \\ ${ }^{4}$ Physics Department, Nelson Mandela University, Port Elizabeth, South Africa
}

\begin{abstract}
Hydrostatic pressure can be used as a powerful diagnostic tool to enable the study of lattice dynamics, defects, impurities and recombination processes in a variety of semiconductor materials and devices. Here we report on intermediate band GaAs solar cells containing GaSb quantum rings which exhibit a 15\% increase in open-circuit voltage under application of 8 kbar hydrostatic pressure at room temperature. The pressure coefficients of the respective optical transitions for the GaSb quantum rings, the wetting layer and the GaAs bulk, were each measured to be $\sim 10.5 \pm 0.5 \mathrm{meV} / \mathrm{kbar}$. A comparison of the pressure induced and temperature induced bandgap changes highlights the significance of the thermal energy of carriers in intermediate band solar cells.
\end{abstract}

\section{Introduction}

Intermediate band solar cells (IBSC) have the potential to achieve a high power conversion efficiency of $63 \%$ compared with $41 \%$ for a single junction solar cell under maximum solar concentration [1, 2]. However, implementing effective two photon absorption and photocarrier generation ideally requires effective de-coupling of a partially filled intermediate band from the conduction and valence bands $[3,4]$. With this aim, GaSb/GaAs quantum ring solar cells (QR SC) have been realized, where the type-II band alignment provides longer carrier lifetime which enhances the extracted photocurrent [5], so that larger short-circuit current density can be obtained compared with the type I-InAs/GaAs system [6]. However, due to reduced electron-hole overlap, the absorption of type-II structures is somewhat lower. Although IBSC are expected to lead the third generation of solar cells, as yet there is no experimental evidence of high efficiency in such devices based upon quantum dots, primarily because the introduction of quantum dots into the depletion region of the solar cell has been found to result in a degradation of the open-circuit voltage [5, 6, 7, 8, 9, 10]. Extensive research is underway to improve both the growth of the quantum dot arrays and also in developing and designing new characterization set-ups for understanding the limitations of these solar cells [11, 12, 13, 14, 15]. Two photon photocurrent and photovoltage measurements have been performed at low temperatures, and bias light power dependence experiments have explored the effect of intermediate band (IB) photo-filling [13]. Complete recovery of the open-circuit voltage, to that in the host material, can be achieved using a combination of high solar concentration and low temperatures [14, 15]. Under these conditions the rate of optical excitation of holes exceeds the rate of thermal escape of holes from the quantum rings which is necessary to achieve effective two photon operation of the intermediate band [16]. 
Hydrostatic pressure has been extensively used in the literature for studying lattice dynamics, defects, impurities and recombination processes in a variety of semiconductor materials and devices including lasers, LEDs and photodetectors [17, 18, 19, 20, 21, 22]. Hydrostatic pressure increases the bandgap of a semiconductor material at a fixed temperature, so that a single device can be tuned under pressure to operate at different wavelengths in a reversible and non-destructive manner. This saves fabricating new devices for each particular study and avoids any issues associated with growth-related changes in material quality. In this work, we present an investigation of $\mathrm{GaSb} / \mathrm{GaAs} \mathrm{QR}$ SC under hydrostatic pressure, where the transition energies increase with application of pressure without modifying the thermal distribution of the carriers. These results are compared with the effect of changing the cell temperature over the same photon energy range, from 1.42 to $1.51 \mathrm{eV}$. By measuring the spectral response we obtain the pressure coefficients for each of the main absorption edges corresponding to the different layers used in the QR SCs: bulk GaAs, GaSb wetting layer (WL) and GaSb quantum ring. We observed an increase in the open-circuit voltage and a decrease in the dark current as the applied pressure increases.

\section{Experimental details}

The samples investigated in this study were p-i-n GaAs based solar cells, containing GaSb quantum rings in the intrinsic region to form the intermediate band, and were grown by molecular beam epitaxy (MBE) on $n+$ GaAs substrates following our earlier design [4] as shown in Fig. 1. The reference solar cell is a p-i-n junction based on GaAs. The structure consists of a $3 \mu \mathrm{m}$ thick n- type $10^{17} \mathrm{~cm}^{-3}$ base layer, followed by a $500 \mathrm{~nm}$ thick intrinsic region and a $500 \mathrm{~nm}$ thick p-type $\left(10^{18} \mathrm{~cm}^{-3}\right)$ emitter layer. In order to improve the device performance, a $50 \mathrm{~nm}$ thick, p-type $\left(10^{18} \mathrm{~cm}^{-3}\right) \mathrm{Al}_{0.8} \mathrm{Ga}_{0.2} \mathrm{As}$ window layer was used, capped with $5 \mathrm{~nm}$ of p-type GaAs followed by a contact layer of $50 \mathrm{~nm}$ thick, heavily doped, p-type $\left(10^{19} \mathrm{~cm}^{-3}\right)$ GaAs. The quantum ring solar cell (QR SC) is of similar construction but contains 10 layers of GaSb quantum rings (QR) capped by $40 \mathrm{~nm}$ of GaAs grown within the intrinsic region of the device. The QRs are grown by the Stranski-Krastanov technique, where the large mismatch of $7 \%$ between GaSb and GaAs initially results in the formation of a thin wetting layer followed by GaSb quantum dots with a density of $\sim 10^{10} \mathrm{~cm}^{-2}$. Following the subsequent deposition of the GaAs capping layer the As-Sb exchange mechanism modifies their shape into QRs [23]. The resulting structures were fabricated into $1 \mathrm{~mm}$ diameter mesaetched solar cells using standard GaAs processing technology. Metallization for the top ptype contact layer was $\mathrm{Au} / \mathrm{Zn} / \mathrm{Au}(10 / 10 / 200 \mathrm{~nm})$ and the bottom n-type was InGe/Au $(20 / 200 \mathrm{~nm})$ to provide good ohmic contacts.

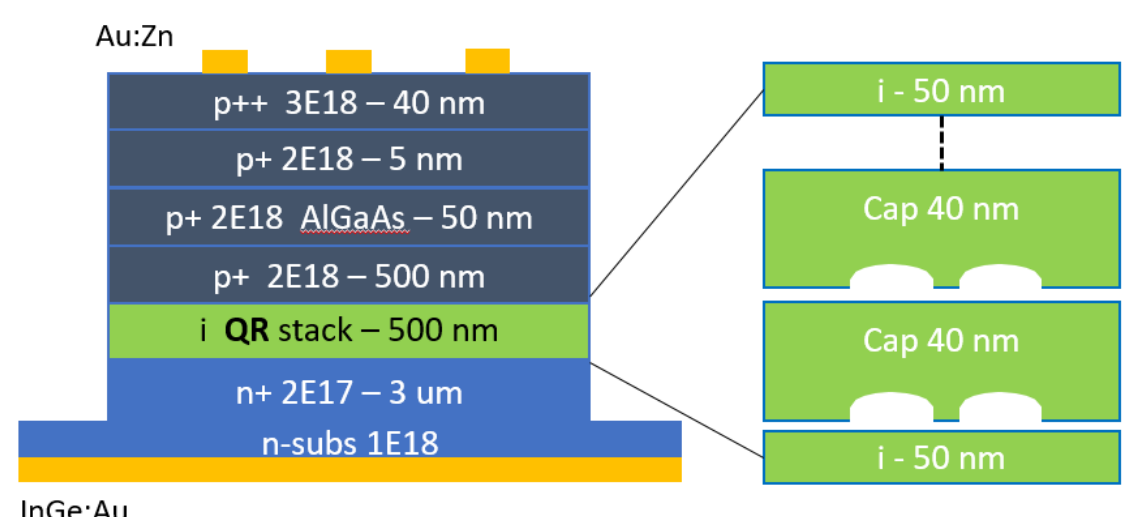


Fig. 1. Structure of the GaAs based QRSC with 10 layers of GaSb QR spaced by $40 \mathrm{~nm}$ in the active region.

Hydrostatic pressure measurements were carried out at room temperature using a Unipress He-gas compressor system capable of generating pressures of up to $10 \mathrm{kbar}$ in a pressure cell where the solar cells were inserted. Electrical access is provided from one side of the pressure cell and the light is incident through a sapphire window from the other side. Further details of the set-up are given in ref. [24]. For this experiment dark and illuminated I-V curves were performed under a maximum applied He pressure of 8 kbar. The spectral response was measured from 400 to $1300 \mathrm{~nm}$ by using a Bentham quartz-halogen (QH) lamp connected to a Bentham TMC-300 grating monochromator. A Ge reference detector was used to obtain the spectral flux dependence of the source. The electrical characterization was performed using a Keithley 2635 source-measure unit, both in the dark and under illuminated conditions, using the QH lamp to simulate solar illumination.

\section{Results and discussion}

a)

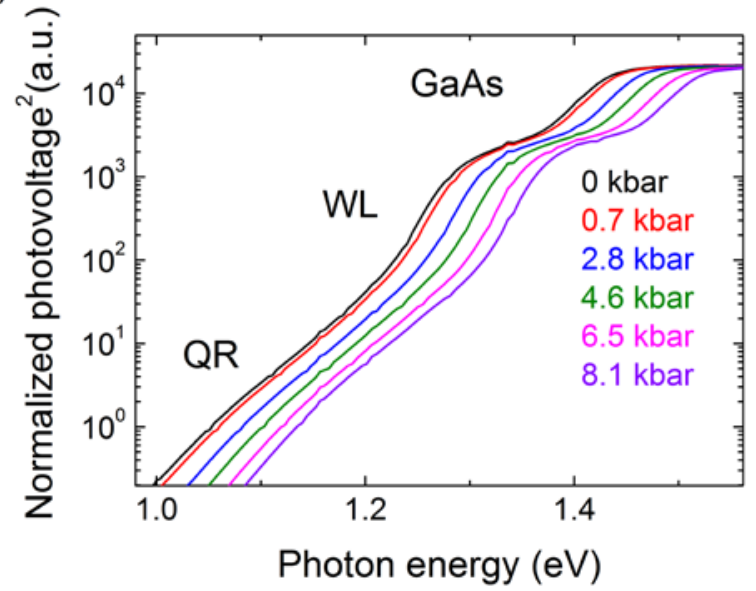

b)

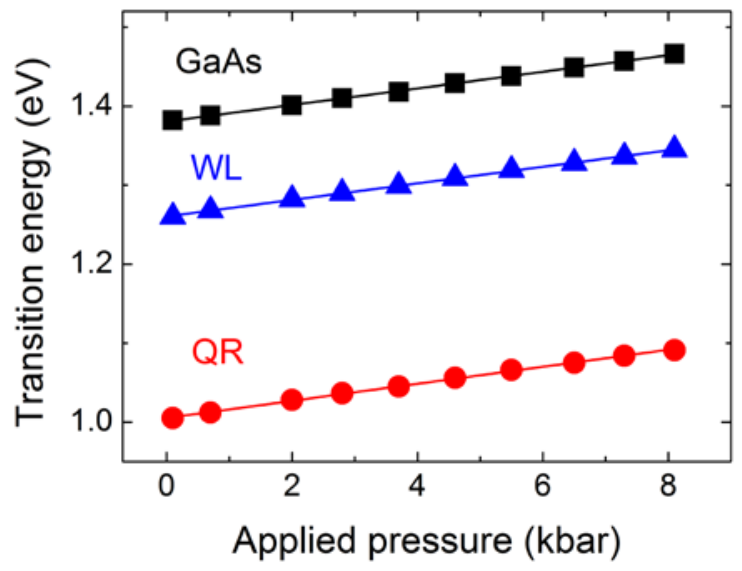

Fig. 2. (a) Square of the normalized photovoltage versus incident photon energy for the $\mathrm{GaSb} / \mathrm{GaAs}$ QRSC for different applied pressures measured at $300 \mathrm{~K}$. Three absorption features can be identified corresponding to: GaAs bulk layer, GaSb wetting layer (WL) and GaSb quantum ring (QR). (b) Transition energy versus applied pressure for the GaAs bulk layer (black squares), GaSb WL (blue triangles) and QR (red circles). The pressure coefficients are obtained from the linear fits (solid lines).

Figure 2(a) shows the square of the normalized photovoltage versus photon energy for the QR SC. Three main absorption features can be identified corresponding to transitions: GaAs valence band to conduction band ( 1.4 eV), GaSb wetting layer (WL) valence band to GaAs conduction band $(\sim 1.25 \mathrm{eV})$, and GaSb QR valence band to GaAs conduction band ( 1.0 $\mathrm{eV}$ ). The pressure dependence of each of these transitions is shown in Figure 2(b). Using a linear fit of the data, the pressure coefficients $(P C)$ were obtained as $10.5 \pm 0.5 \mathrm{meV} / \mathrm{kbar}$ for the GaAs bulk layer feature, which is in agreement with literature values, which lie in the range 8.5-12.6 meV/kbar [25] and $10.5 \pm 0.5 \mathrm{meV} / \mathrm{kbar}$ for both the GaSb WL and QR transitions.

Using the measured pressure coefficients $(P C)$ for each transition from Figure 2(b), together with the deformation potential values for conduction band $\left(a_{c}\right)$ and valence band edges $\left(a_{v}\right)$ shown in Table I, we obtained the $300 \mathrm{~K}$ band diagram of the GaSb QR under 0 and $8 \mathrm{kbar}$ of 
applied pressure shown in Fig. 3. The induced shift in the conduction band $(\Delta E c)$ under a certain range of pressure $(\Delta P)$ is calculated using Eq. 1

$$
\Delta E C=\Delta P \frac{a_{c}}{a_{c}+a_{v}} P C
$$

where the valence band shift is obtained using a similar expression [26].

Table I. Deformation potential values for conduction and valence band edges obtained from literature [25, 27].

\begin{tabular}{|l|l|l|}
\hline & GaAs bulk & GaSb bulk \\
\hline$a_{c}\left(\mathrm{meV}^{-1}\right)$ & 7.17 & 7.5 \\
\hline$a_{v}\left(\mathrm{meV}^{-1}\right)$ & 1.16 & 0.8 \\
\hline
\end{tabular}

The confinement energy of the QR levels at 0 kbar, which depends on size, shape and charge, is known to be $400 \pm 200 \mathrm{meV}$ from photoluminescence and spectral response measurements $[28,29,30]$. At the Brillouin zone centre $(\Gamma)$, the conduction band has a much stronger pressure dependence than the valence band $\left(a_{c}>>a_{v}\right)$, hence the change in the valence band offset between $\mathrm{GaAs}$ and $\mathrm{GaSb}$ is negligible, whereas the conduction band offset increases by $30 \mathrm{meV}$ at $8 \mathrm{kbar}$. Since the QR confinement energy is therefore expected to remain approximately constant with changes in pressure, we expect no change in the thermal emission rate of holes from the QR under illumination at $300 \mathrm{~K}$. We can, however, expect that the overall increase in energy gap with applied pressure will decrease the dark current and increase the open-circuit voltage of the solar cell.

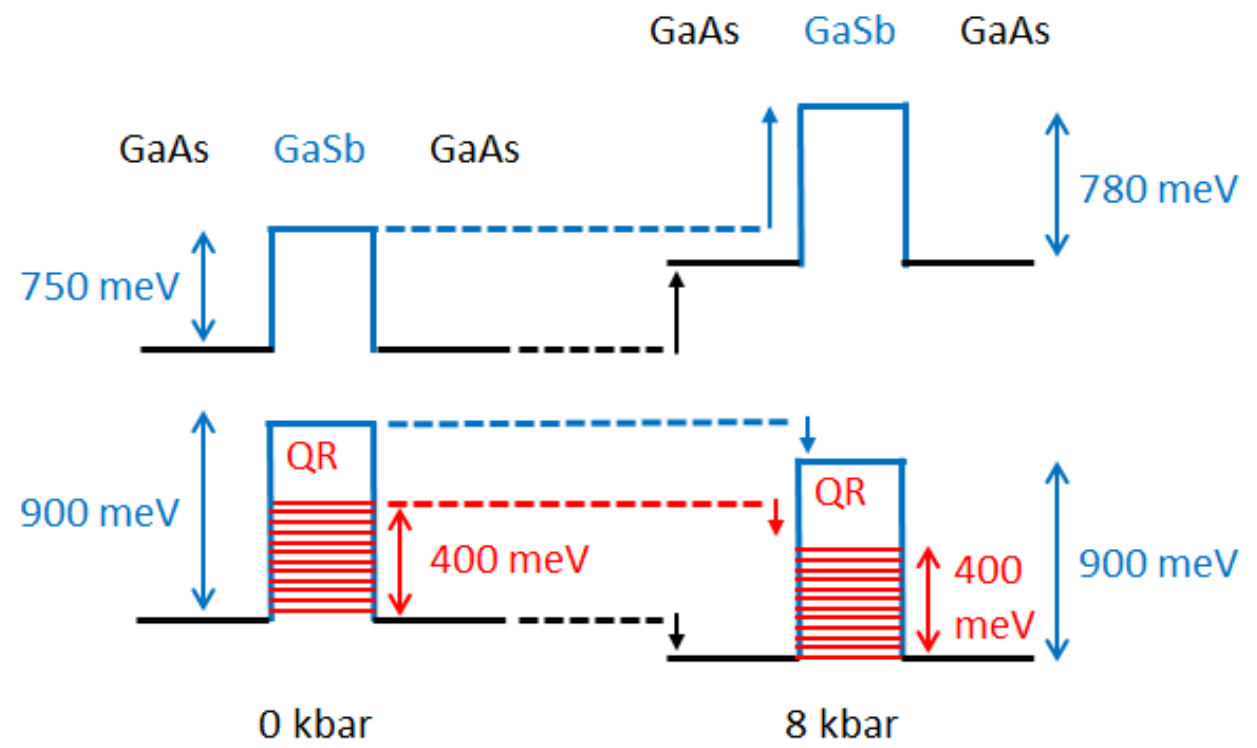

Fig. 3. A schematic energy band diagram for GaSb/GaAs QR at $300 \mathrm{~K}$ under 0 and 8 kbar of hydrostatic pressure. Wetting layer energy levels are not shown for simplicity.

Figure 4(a) shows a logarithmic scale plot of the dark current density-voltage $(J-V)$ curve for the QR SC at $300 \mathrm{~K}$ measured in forward bias at different hydrostatic pressures in the range 0 to 8 kbar. The central linear region can be fitted using the Shockley diode equation 


$$
J=J_{0}\left[\exp \left(\frac{e V}{n k T}\right)-1\right]
$$

where $T$ is the cell temperature, $k$ the Boltzman constant, $e$ the electron charge, $J_{o}$ is the dark diode saturation current and $n$ is the ideality factor. Eq. 2 gives an ideality factor of between $n=1.70$ and 1.83 for the QR SC, whereas for the reference cell $n$ remains almost constant around 2.40. The dark current density decreases as the applied pressure increases, which is shown in Fig. 4(b) for both the QR SC and the GaAs reference cell. The slopes of the linear fits to the data are the same within uncertainties and both reduce with increasing pressure at a rate of roughly $0.15 \mathrm{mAcm}^{-2} / \mathrm{kbar}$. The pressure dependence of the dark current and ideality factor originates mainly from the reduction in the intrinsic carrier concentration, $n_{i}$, associated with the increase in the bandgap energy, which affects the saturation current density, given by

$$
J_{0}=q L_{N}\left(\frac{n_{i}^{2} / N_{A}}{\tau_{n}}\right)+q L_{P}\left(\frac{n_{i}^{2} / N_{D}}{\tau_{p}}\right)+\frac{q n_{i} W}{\tau_{n} \tau_{p}}
$$

where $L_{N}, L_{P}$ and $\tau_{n}, \tau_{p}$ are the minority carrier diffusion lengths and lifetimes for electrons and holes, respectively, $N_{A}$ and $N_{D}$ are the p- and n-type doping concentrations, and $n_{i}$ is the intrinsic carrier concentration, which depends exponentially on the bandgap energy. For GaAs based solar cells recombination current is dominant over diffusion, so that $J_{0}$ depends linearly on $n_{i}$. However, in QR SC with an ideality factor between 1 and 2 both diffusion and recombination currents are present. Following Eq. 3, when $n_{i}$ decreases under pressure diffusion current reduces quicker than recombination as it depends on the square of $n_{i}$, resulting in an ideality factor closer to 2 . 
a)

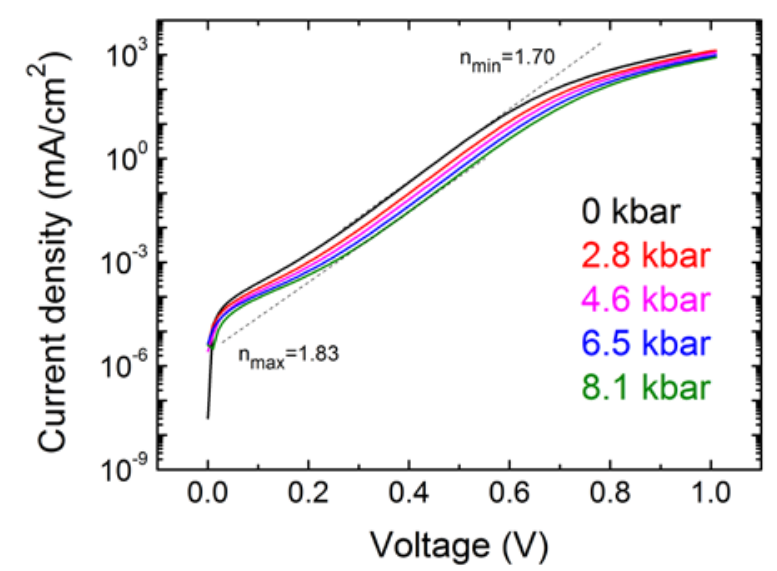

c)

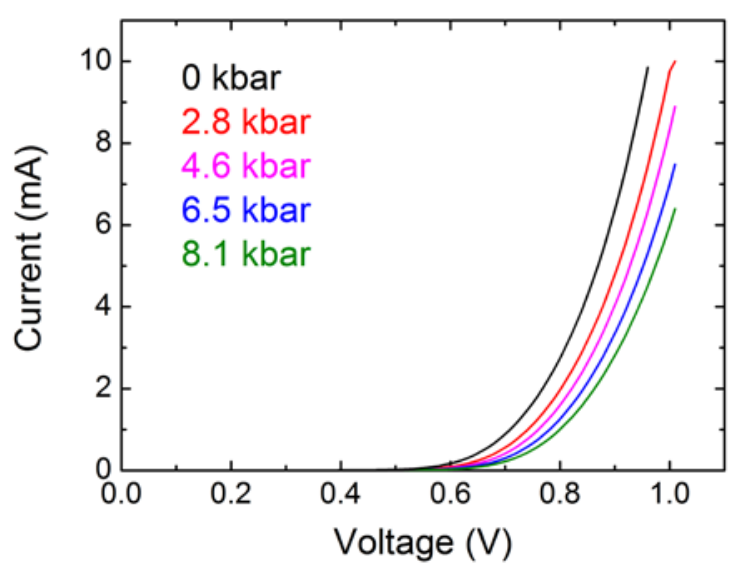

b)

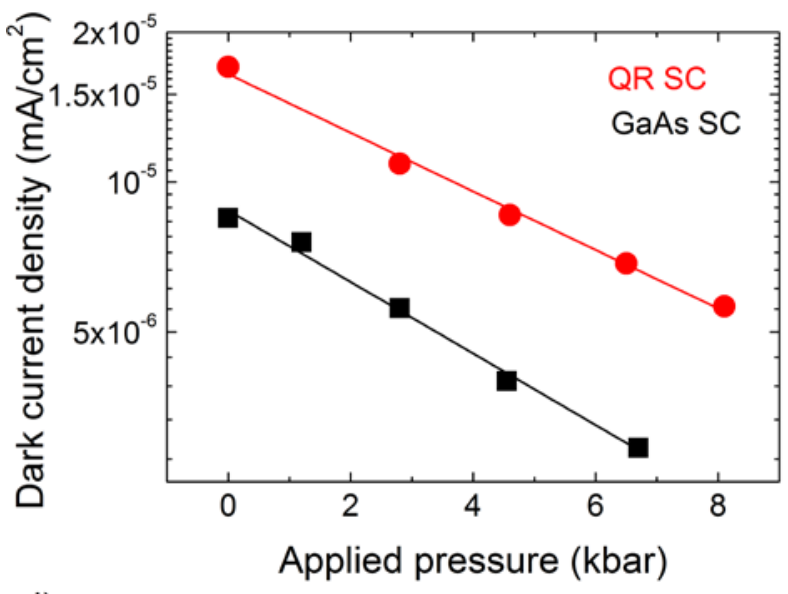

d)

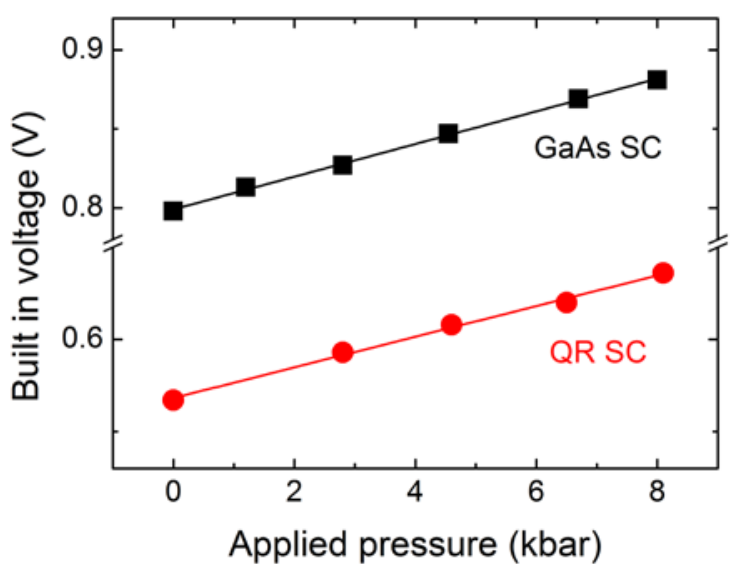

Fig. 4. (a) Dark current density (J-V) characteristics in forward bias under hydrostatic pressure for the QR SC. (b) Logarithmic scale plot of saturation dark current density, $J_{o}$, versus pressure for the QR SC (red circles) and GaAs SC (black squares). (c) The current-voltage (I-V) characteristics for the QR SC measured at increasing pressure. (d) Solid points are the experimental values and the lines are the linear fits of the built-in voltage for the QR SC (red circles) and GaAs control (black squares) cell extracted from the corresponding (I-V) plots.

Figure 4(c) shows the linear current-voltage (I-V) characteristics in the dark, measured from the QR SC for different applied pressures. The built-in (knee) voltage (obtained by extrapolation) increases with applied pressure as expected, again following the increase in the GaAs bandgap. Figure 4(d) shows a comparison of the built-in voltage against applied pressure for the QR SC and the control cell. The corresponding pressure coefficients were extracted as $(8.3 \pm 0.5) \mathrm{mV} / \mathrm{kbar}$ for the QR SC and $(10.4 \pm 0.5) \mathrm{mV} / \mathrm{kbar}$ for the control cell respectively. These are approximately in agreement with the bandgap pressure dependence values obtained from the spectral characterization in Fig. 1.

Figure 5(a) shows the illuminated J-V curves for the QR SC as a function of pressure. The short-circuit current, $J_{S C}$, decreases as the pressure increases, whereas the open-circuit voltage, $V_{O C}$, increases with pressure. This behaviour is expected as the bandgap energy is increasing. The corresponding changes in $J_{S C}$ and $V_{O C}$ for the QR SC and the GaAs control cell are shown for comparison in Figures 5(b) and 5(c), respectively. The resulting pressure coefficients obtained from these measurements are given in Table II. Within the experimental error, there is no difference in the pressure dependence of $J_{S C}$ for the QR SC and the GaAs 
control cell, because the photocurrent is mainly produced from the GaAs bulk material (which has the same pressure dependence in both samples). The open-circuit voltage is expected to increase following the bandgap and we obtained an increase of $15 \%$ in the $V_{O C}$ of the QR SC over the $8 \mathrm{kbar}$ pressure range. The output voltage is degraded when QRs are introduced due to the increase in recombination through the $\mathrm{QR}$ energy levels and/or associated defect levels. By applying pressure, we did not observe any significant difference in recombination in the QR SC compared with the GaAs control cell. The solid lines in Figure 5(c) are linear fits to the data using the equation for open-circuit voltage as a function of pressure

$$
V_{o c}=\frac{n k T}{q} \ln \left(\frac{J_{S c}}{J_{0}}+1\right)
$$

where the pressure dependence of $n, J_{0}$ and $J_{S C}$ have been obtained from Figures 4(a), (b) and Figure 5 (b) respectively, showing that the measurements are self-consistent.

a)

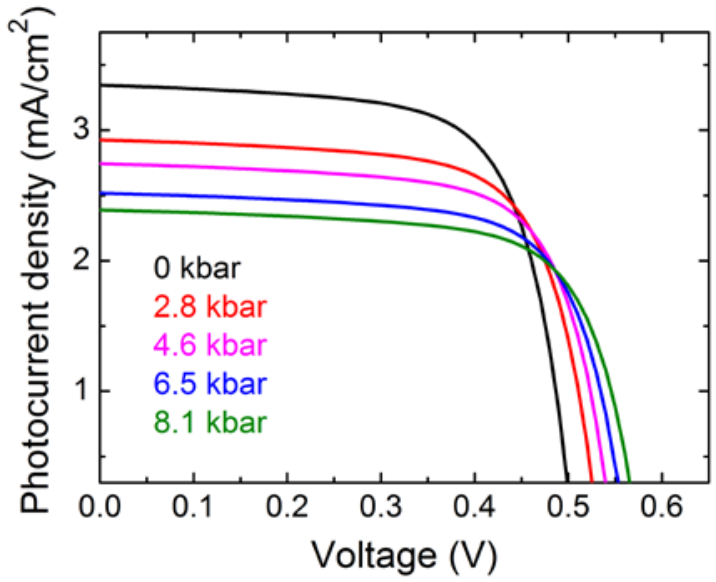

b)

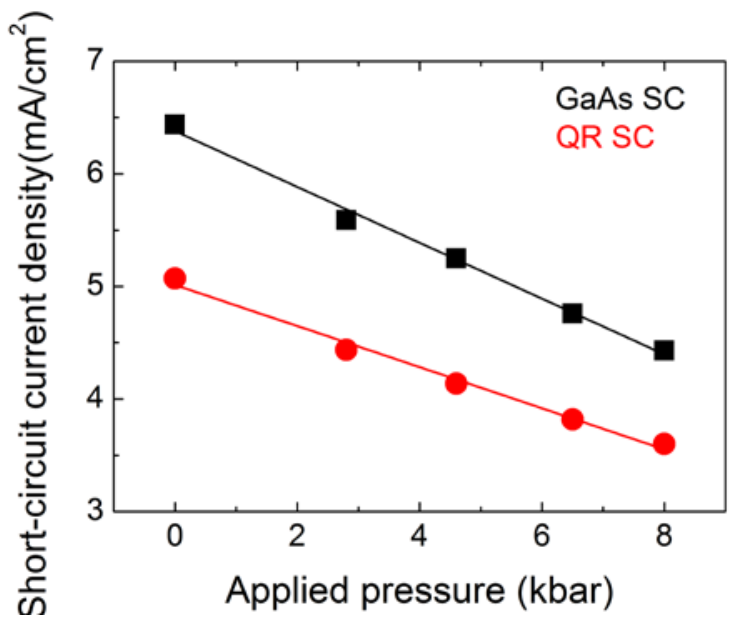

c)

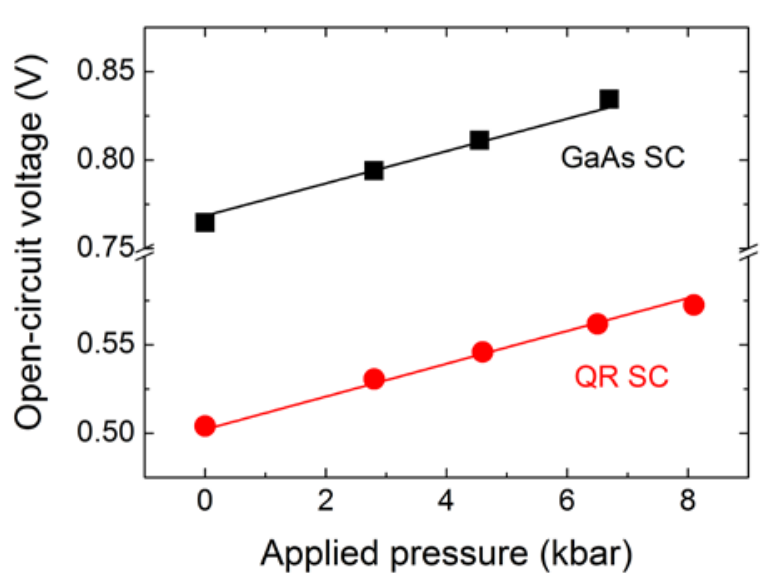

Fig. 5. (a) J-V characteristics of the QRSC under QH lamp illumination as the hydrostatic pressure increases up to 8 kbar. (b) Short-circuit current density versus pressure for the GaAs SC (black squares) and QR SC (red circles), showing $J_{S C}$ decreasing as the pressure increases for both samples. (c) Open-circuit voltage versus pressure for the two cells, the solid lines joining the data points are linear fits of the experimental data using Eq. 4. 
Table II. A comparison of the $300 \mathrm{~K}$ pressure dependence of the open-circuit voltage $\left(V_{O C}\right)$, the built-in voltage $\left(V_{b i}\right)$, the short-circuit current density $\left(U_{S C}\right)$ and the dark current density $\left.\mathrm{Ln}\left[J_{0}\right]\right)$ for the QRSC and GaAs solar cell over the 0 to 8 kbar range.

\begin{tabular}{|c|c|c|c|c|}
\hline $\begin{array}{c}\text { Pressure } \\
\text { dependence }\end{array}$ & $\begin{array}{c}V_{O C} \\
(\mathrm{mV} / \mathrm{kbar})\end{array}$ & $\begin{array}{c}V_{b i} \\
(\mathrm{mV} / \mathrm{kbar})\end{array}$ & $\begin{array}{c}J_{S C} \\
\left(\mathrm{mAcm}-{ }^{2} / \mathrm{kbar}\right)\end{array}$ & $\begin{array}{c}\mathrm{Ln}\left[J_{0}\right] \\
\left(\mathrm{mAcm}^{-2} / \mathrm{kbar}\right)\end{array}$ \\
\hline QRSC & $8.5 \pm 0.5(15 \%)$ & $8.3 \pm 0.5$ & $0.18 \pm 0.02$ & $0.14 \pm 0.02$ \\
\hline GaAs SC & $9.3 \pm 0.5(9 \%)$ & $10.4 \pm 0.5$ & $0.25 \pm 0.04$ & $0.16 \pm 0.02$ \\
\hline
\end{tabular}

a)

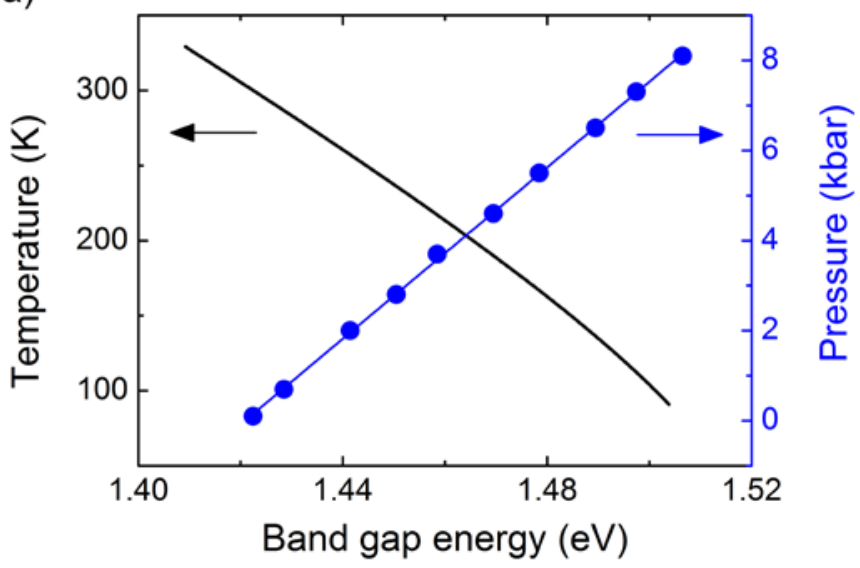

b)

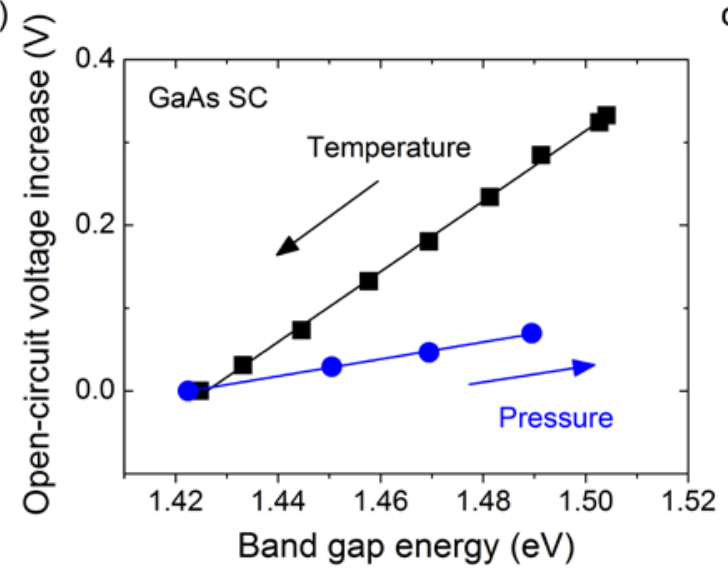

c)

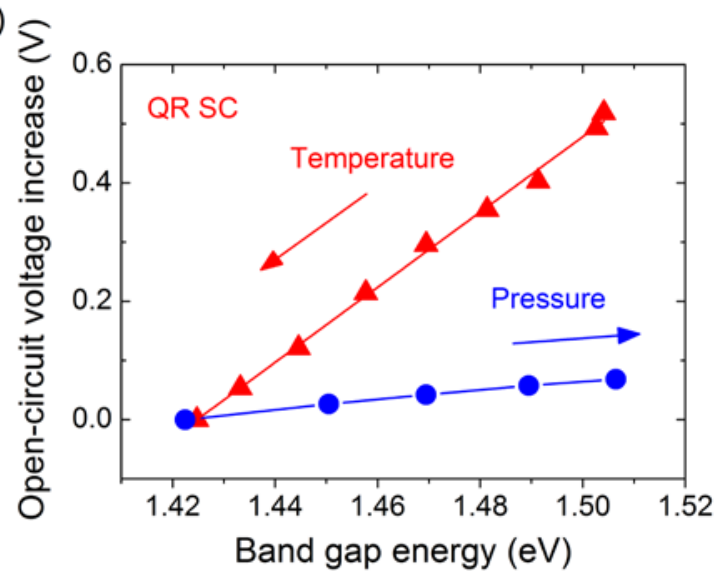

Fig. 6. (a) Energy gap as a function of temperature for GaAs SC (solid black line) based on the Varshni empirical law [31] and the experimental pressure dependence for GaAs SC (blue circles) measured in this work. (b) The open-circuit voltage increase of the GaAs reference cell; dependence on temperature (black squares) and pressure (blue circles). (c) The corresponding changes in opencircuit voltage for the QR SC with temperature (red triangles) and pressure (blue circles).

Finally, we compare the effect of applying hydrostatic pressure to the temperature dependence of our solar cells. Either increasing pressure or reducing temperature can increase the energy gap decreasing the recombination in GaAs and QR levels, but cooling down will change the carrier thermal distribution as well.

By studying the pressure dependence, we can separate the different contributions and isolate the effect of modifying only the transition energy (bandgap change without thermal effects). 
Figure 6(a) shows a comparison of the dependence of the GaAs energy gap with temperature and pressure. In order to represent the temperature dependence, we have used the Varshni empirical fit of a GaAs SC obtained from our previous work [32] over the range from 90 to $300 \mathrm{~K}$. For the pressure dependence, we use the results presented in Figure 2(b) for the GaAs and QR SC covering the range from 0 to $8 \mathrm{kbar}$. In each case the change in bandgap is roughly similar $\sim 100 \mathrm{meV}(4 \mathrm{kT})$. Figure 6 (b) shows the corresponding increase in $V_{O C}$ for the control cell, while Figure 6(c) shows the $V_{O C}$ increase for the QR SC. In both cases reducing the temperature has the strongest influence compared to applying pressure and is more effective in increasing $V_{O C}$ in the QR SC than in the GaAs cell. Recombination and consequently dark current depends directly on $n_{i}$. Applying a maximum pressure of $8 \mathrm{kbar}$ reduces $n_{i}$ by a factor of 5 , however reducing the temperature to $90 \mathrm{~K}$ reduces $n_{i}$ effectively to zero. In QR SC the $V_{O C}$ is limited by recombination through $\mathrm{QR}$ energy levels. Consequently, an increase in $V_{O C}$ means a reduction in recombination between holes trapped inside the QRs and free electrons in the CB. In the case of applying pressure, the reduced recombination is dominated by $n_{i}$ in GaAs CB as both QR SC and GaAs SC follow the same trend under pressure. However, as reported earlier in Ref. 32, when decreasing temperature $V_{O C}$ recovers in QR SC meaning that recombination through QRs is further suppressed compared to GaAs SC. In summary, by reducing $n_{i}$ the recombination through confined holes within the GaSb quantum rings reduces resulting in an increase of $V_{O C}$ by $15 \%$ under $8 \mathrm{kbar}$ and a recovery of $50 \%$ at $90 \mathrm{~K}$. This experimental work provides a useful way to study recombination in solar cell devices and isolate the contribution of the thermal carrier distribution from widening the bandgap of the host material in intermediate band solar cells.

\section{Conclusion}

We have investigated the effect of applying hydrostatic pressure to GaAs intermediate band solar cells containing GaSb quantum rings and compared the increase in open-circuit voltage to that obtained with decreasing the temperature. Our GaSb/GaAs QR SC exhibits an output voltage increase of $15 \%$ in response to applying a hydrostatic pressure of $8 \mathrm{kbar}$ as intrinsic carrier concentration is reduced by a factor 5 . The pressure coefficient was similar to that of the control cell; being $(8.5 \pm 0.5$ and $9.3 \pm 0.5) \mathrm{meV} / \mathrm{kbar}$ for the QR SC and the reference GaAs SC, respectively. The dark current and the short-circuit current under QH lamp illumination both decreased in response to increasing pressure following the increase in the bandgap energy. The pressure coefficients of the bandgap variation with pressure were found to be in agreement with those previously reported in the literature. Using hydrostatic pressure we were able to investigate the effect of modifying the transition energy independently from the thermal carrier distribution. We observed that temperature has the strongest influence on the open-circuit voltage compared to hydrostatic pressure and is more effective in increasing $V_{O C}$ in the QR SC than in the GaAs cell. This indicates that it is more important to control the thermal distribution of carriers than the overall bandgap in developing these type-II intermediate band solar cells.

\section{References}

[1] A. Luque and A. Martí, Phys. Rev. Lett., vol. 78, p. 5014-5017, 1997. 
[2] A. Luque, A. Marti and C. Stanley, Nat. Photonics, vol. 6, p. 146-152, 2012.

[3] R. Strandberg and T. Reenaas, IEEE T Electron Dev, vol. 58, p. 2559-2565, 2011.

[4] P. J. Carrington, M. C. Wagener, J. R. Botha, A. M. Sanchez and A. Krier, Appl. Phys. Lett., vol. 101, 2012.

[5] C. G. Bailey, D. V. Forbes, S. J. Polly, Z. S. Bittner, Y. Dai, C. Mackos, R. P. Raffaelle and S. M. Hubbard, IEEE J. Photovoltaics, vol. 2, p. 269-275, 2012.

[6] W. Liu, H. Wu, F. Tsao, T. Hsu and J. Chyi, Sol. Energy Mater. Sol. Cells, vol. 105, p. 237-241, 2012.

[7] S. M. Hubbard, C. D. Cress, C. G. Bailey, R. P. Raffaelle, S. G. Bailey and D. M. Wilt, Appl. Phys. Lett., vol. 92, $\mathrm{n}^{\mathrm{o}}$ 12, p. 123512, 2008.

[8] F. K. Tutu, I. R. Sellers, M. G. Peinado, C. E. Pastore, S. M. Willis, A. R. Watt, T. Wang and H. Y. Liu, Journal of Appl. Phys., vol. 111, nº 4, p. 046101, 2012.

[9] T. Sugaya, O. Numakami, R. Oshima, S. Furue, H. Komaki, T. Amano, K. Matsubara, Y. Okano and S. Niki, Energy Environ. Sci., vol. 5, p. 6233-6237, 2012.

[10] R. B. Laghumavarapu, A. Moscho, A. Khoshakhlagh, M. El-Emawy, L. F. Lester and D. L. Huffaker, Appl. Phys. Lett., vol. 90, p. 173125, 2007.

[11] N. S. Beattie, P. See, G. Zoppi, P. M. Ushasree, M. Duchamp, I. Farrer, D. A. Ritchie and S. Tomić, ACS Photonics, vol. 4, $\mathrm{n}^{\circ}$ 11, pp. 2745-2750, 2017.

[12] M. C. Wagener, D. Montesdeoca, A. R. J. Marshall, A. Krier, J. R. Botha and P. J. Carrington, Sol. Energy Mater. Sol. Cells, vol. (submitted), 2018.

[13] I. Ramiro, J. Villa, C. Tablero, E. Antolín, A. Luque, A. Martí, J. Hwang, J. Phillips, A. J. Martin and J. Millunchick, Phys. Rev. B, vol. 96, p. 125422, 2017.

[14] P. G. Linares, A. Marti, E. Antolin, I. Ramiro, E. Lopez, C. D. Farmer, C. R. Stanley and A. Luque, IEEE Journal of Photovoltaics, vol. 3, p. 031299, 2013.

[15] P. G. Linares, A. Marti, E. Antolin, C. D. Farmer, I. Ramiro, C. R. Stanley and A. Luque, Sol. Energy Mater. Sol. Cells, vol. 98, $n^{\circ}$ 240, 2012.

[16] J. Hwang, PhD thesis University of Michigan, 2013.

[17] W. Paul and D. M. Warschauer, Journal of Phys. and Chem. of Solids, vol. 6, n ${ }^{\circ}$ 6, 1958.

[18] M. I. Daunov, I. K. Kamilov and S. F. Gabibov, Semicond. Sci. Technol., vol. 16, nº 511, 2001.

[19] T. Higashi, S. J. Sweeney, A. F. Phillips, A. R. Adams, E. P. O’Reilly, T. Uchida and T. Fujii, IEEE Journal of Selected Topics in Quant. Electr., vol. 5, n 3, p. 413, 1999.

[20] I. P. Marko, A. D. Andreev, A. R. Adams, R. Krebs, J. P. Reithmaier and A. Forchel, IEEE Journal of Selected Topics in Quant. Electr., vol. 9, p. 1300, 2003. 
[21] N. Hossain, I. P. Marko, S. R. Jin, K. Hild, S. J. Sweeney, R. B. Lewis, D. A. Beaton and T. Tiedje, App. Phys. Lett., vol. 100, p. 051105, 2012.

[22] B. G. Crutchley, I. P. Marko, J. Pal, M. A. Migliorato and S. J. Sweeney, Phys. Status Solidi B, vol. 250, no 4, p. 698, 2013.

[23] E. P. Smakman, J. K. Garleff, R. J. Young, M. Hayne, P. Rambabu and P. M. Koenraad, App. Phys. Lett., vol. 100, p. 142116, 2012.

[24] S. R. Jin, S. J. Sweeney, C. N. Ahmad, A. R. Adams and B. N. Murdin, App. Phys. Lett., vol. 85, $\mathrm{n}^{\circ} 3$, p. 357, 2004.

[25] S. H. Wei and A. Zunger, Phys. Rev. B, vol. 60, n 8, p. 5404, 1999.

[26] W. Paul, High Pressure in Semiconductor Physics II, vol. 55, Academic Press, 1998.

[27] I. Vurgaftman, J. R. Meyer and L. R. Ram-Mohan, Journal of App. Phys., vol. 89, n 11, p. 5815, 2001.

[28] T. Nowozin, A. Marent, L. Bonato, A. Schliwa, D. Bimberg, E. P. Smakman, J. K. Garleff, P. M. Koenraad, R. J. Young and M. Hayne, Phys. Rev. B, vol. 86, p. 035305, 2012.

[29] M. C. Wagener, P. J. Carrington, J. R. Botha and A. Krier, App. Phys. Lett., vol. 103, p. 063902 , 2013.

[30] R. Timm, H. Eisele, A. Lenz, L. Ivanova, V. Vosseburger, T. Warming, D. Bimberg, I. Farrer, D. A. Ritchie and M. Dahne, Nano Lett., vol. 10, p. 3972, 2010.

[31] Y. P. Varshni, Physica, vol. 34, p. 149, 1967.

[32] H. Fujita, P. J. Carrington, M. C. Wagener, J. R. Botha, A. R. J. Marshall, J. James, A. Krier, K. Lee and N. J. Ekins-Daukes, Prog. Photovolt., vol. 23, p. 1896, 2015.

Acknowledgements

This work was supported by the EU Marie Sklodowska-Curie Initial Training network (ITN) PROMIS - Postgraduate Research on Dilute Metamorphic Nanostructures and Metamaterials in Semiconductor Photonics Grant agreement No 641899. P.J.C. acknowledges support from the Royal Academy of Engineering through a Research Fellowship grant 10216/114 and the Lancaster University Research Committee. SJS and IM acknowledge the support of EPSRC through grants EP/N021037/1 and EP/H005587/1.

Data is available in: 10.17635/lancaster/researchdata/214 